\title{
Metastatic Osseous Disease Masquerading as Infection, Diagnosed on Bone Scintigraphy and SPECT/CT
}

Gracie A. Keown, Sunderarajan Jayaraman, and Jennifer Davidson

Nuclear Medicine Department, Auckland City Hospital, Auckland District Health Board, Grafton, Auckland, New Zealand

We present a 72-y-old woman with an osseous metastasis to the spine that had an atypical appearance on both nuclear medicine and radiologic modalities and was misdiagnosed as an abscess. We discuss the pitfalls of bone scintigraphy and the causes of photopenic metastatic lesions.

Key Words: bone; scintigraphy; metastases; photopenic

J Nucl Med Technol 2020; 48:179-180

DOI: 10.2967/jnmt.119.232850

D espite its low specificity, bone scintigraphy is one of the most commonly ordered tests in the investigation of suspected skeletal metastases (I). Sclerotic metastases are typically radiopharmaceutical-avid on bone scintigraphy and on SPECT/CT. Metastases may show bone destruction or an extraosseous soft-tissue component (2). This is a case report of an atypical metastasis that was not avid on bone scans and had a large cystic component causing diagnostic uncertainty.

\section{CASE REPORT}

A 72-y-old woman presented with back pain and rightleg radiculopathy. Her past medical history included spindlecell sarcoma of the left thigh treated with radiotherapy and surgery $1 \mathrm{y}$ beforehand.

Contrast-enhanced CT demonstrated a ring-enhancing lesion involving the posterior elements of the right L4 vertebra, with a soft-tissue component extending into the neural foramen and spinal canal.

On MRI, the lesion was of a uniformly low T1 signal. The T2 signal was mixed, with fluid-fluid levels, consistent with intralesional hemorrhage. After gadolinium contrast administration, there was peripheral enhancement and central nonenhancement (Fig. 1).

The patient was afebrile, with a normal white cell count; however, C-reactive protein levels rose from 12 to $175 \mathrm{mg} / \mathrm{L}$. The differential diagnosis was metastatic deposit versus infection.

Received Jul. 31, 2019; revision accepted Oct. 16, 2019.

For correspondence or reprints contact: Gracie A. Keown, Auckland City Hospital, 2 Park Rd., Grafton, Auckland 1023, New Zealand.

E-mail: gracie.keown@gmail.com

Published online Dec. 6, 2019.

COPYRIGHT (c) 2020 by the Society of Nuclear Medicine and Molecular Imaging.
Ultrasound-guided fine-needle aspiration was performed, and histologic examination revealed large numbers of neutrophils and other inflammatory cells, with no malignant cells, diagnostic of a paraspinal abscess.

Despite these results, there was ongoing concern and bone scintigraphy was performed. Whole-body imaging did not show any uptake of ${ }^{99 \mathrm{~m}} \mathrm{Tc}$-methylene diphosphonate ${ }^{99 \mathrm{~m}} \mathrm{Tc}-\mathrm{MDP}$ ) tracer within the lesion (Fig. 2).

The SPECT/CT images showed bony destruction of the right L4 pedicle, a typical location for a metastasis (Fig. 3). Nuclear medicine findings played an important role by identifying the photopenic lesion associated with lysis and remaining suggestive despite the apparent false-negative whole-body study. This prompted CT-guided core biopsy. Histologic examination revealed malignant spindle cells with marked nuclear atypia and extensive necrosis, consistent with metastatic sarcoma. The patient proceeded with palliative radiotherapy.

\section{DISCUSSION}

Bone scans are frequently used to diagnose osseous metastases, with high sensitivity rates of $95 \%$ (3). However, lesions can be photopenic, and the interpreter must be aware of the causes for this. In our case, the highly aggressive, lytic

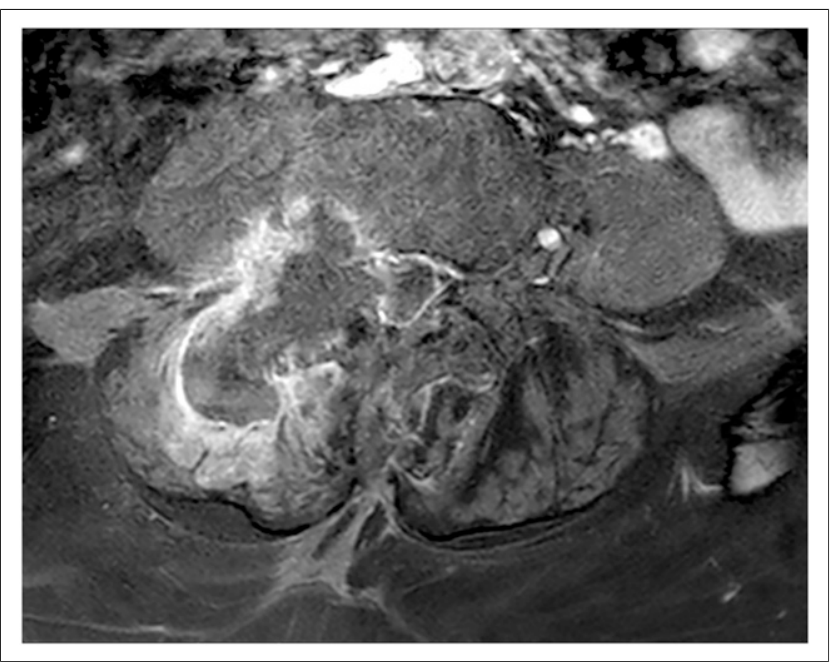

FIGURE 1. T1-weighted contrast-enhanced axial MR image through the lesion demonstrates central nonenhancement. The appearance was initially interpreted as collection; hence, infection was the initially proposed diagnosis. 


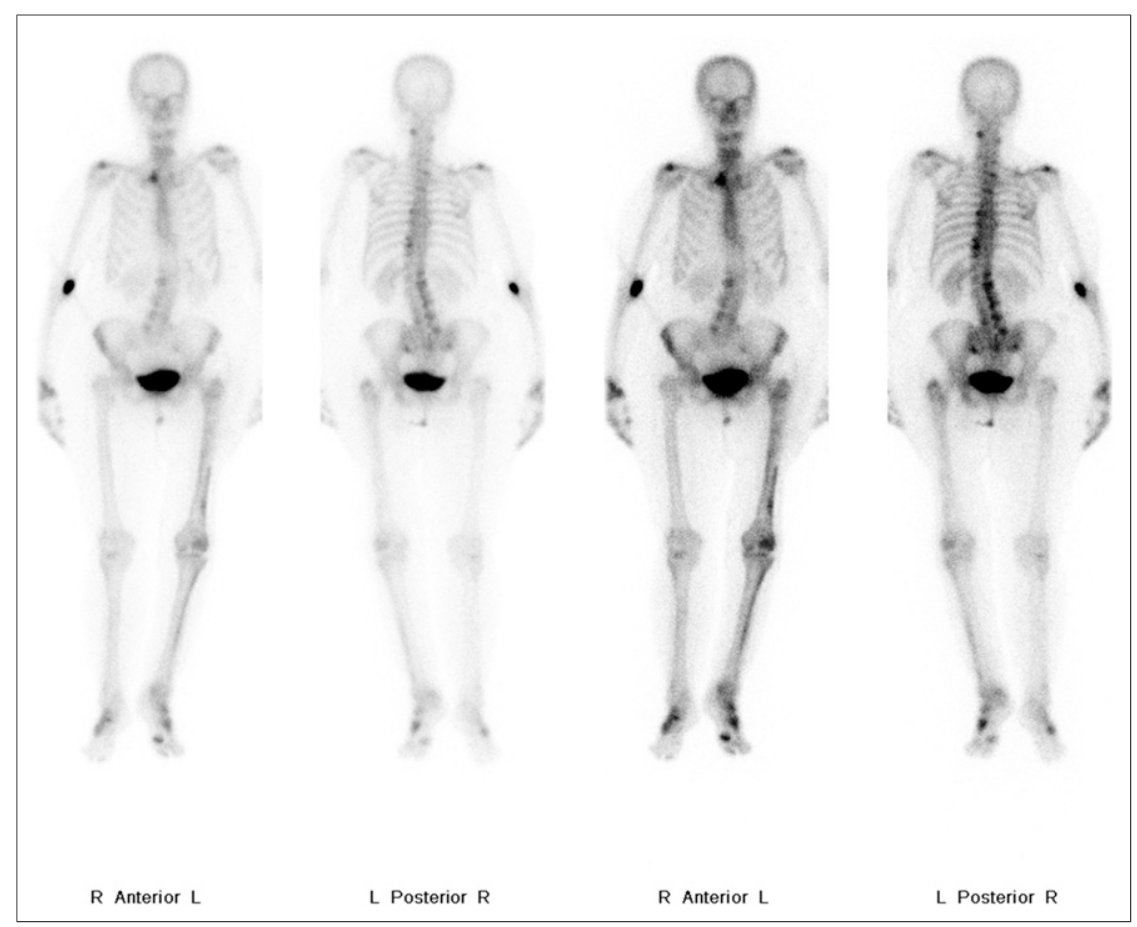

FIGURE 2. Whole-body bone scan demonstrating areas of uptake within the lumbar spine secondary to degenerative change but no tracer uptake in the metastatic lesion. Asymmetric uptake in the left lower limb is due to previous surgery, radiotherapy, and altered biomechanics. significant information toward the overall diagnosis. The case also emphasizes the importance of a thorough review of prior cross-sectional imaging.

A final teaching point this case highlights is the sampling error inherent in fine-needle aspiration. Core biopsy should always be preferentially obtained for conclusive histologic results.

\section{CONCLUSION}

Metastatic osseous disease is frequently investigated with bone scintigraphy. It is important for staff involved in nuclear medicine to be aware of potential pitfalls and causes of falsenegative whole-body imaging. Correlation with SPECT/CT is crucial for detection of associated features of malignancy, particularly in unusual cases.

\section{DISCLOSURE}

No potential conflict of interest relevant to this article was reported.
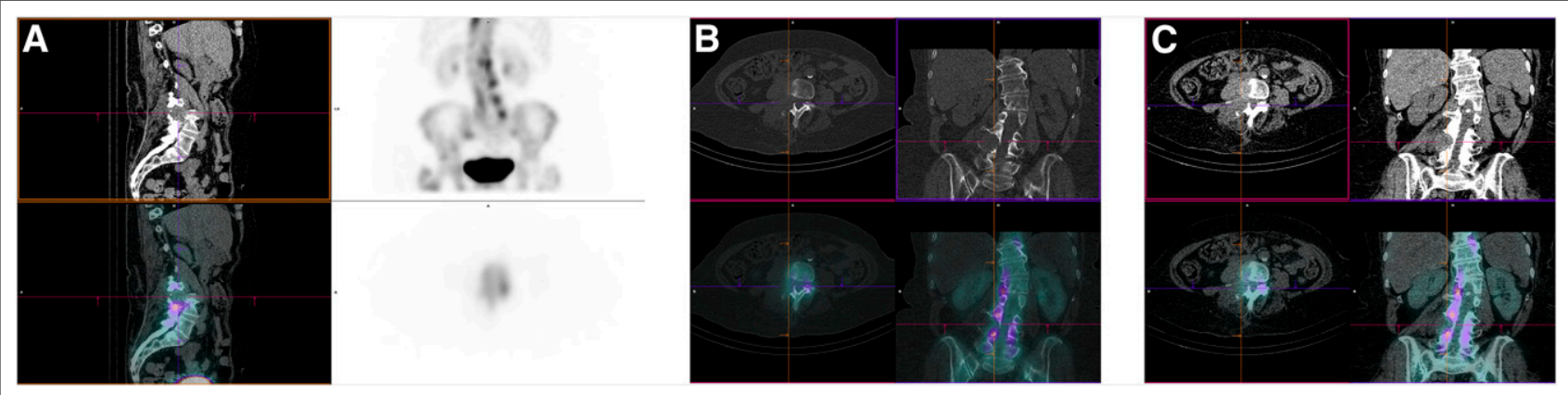

FIGURE 3. SPECT/CT $3 \mathrm{~h}$ after intravenous administration of 99mTc MDP. (A) Images showing that lesion is photopenic; however, soft-tissue mass and associated lysis of right L4 pedicle are evident: sagittal soft-tissue reformat, unenhanced CT (top left); maximum-intensity projection of SPECT/CT (top right); sagittal soft-tissue SPECT/CT (bottom left); axial SPECT acquisition at L4 level (bottom right). (B) Images giving both structural and functional information: axial and coronal bone window CT acquisition (top); axial and coronal bone window SPECT/CT (bottom). (C) images demonstrating extensive soft-tissue involvement of L4 and confirming diagnosis of metastatic sarcoma: axial and coronal CT soft-tissue reformats (top); axial and coronal SPECT/CT (bottom).

nature of the lesion was the likely cause. For aggressive tumors such as this, ${ }^{18} \mathrm{~F}$-FDG PET/CT can play a complementary role and is indicated in staging for various types of sarcomas. Other photopenic osteolytic tumors include hepatocellular, renal, thyroid carcinoma, and tumors that are confined to the bone marrow such as myeloma and lymphoma (4).

The case highlights the importance of SPECT/CT as an adjunct to whole-body imaging, as the CT component adds

\section{REFERENCES}

1. Carmona Echeverria LM, Drudge-Coates L, Wilkins CJ, et al. Bone scan is of doubtful value as a first staging test in the primary presentation of prostate cancer. ISRN Oncol. 2012;2012:585017.

2. Christian PE, Waterstram-Rich KM. Nuclear Medicine and PET/CT. 7th ed. St Louis, MO: Elsevier Mosby; 2012.

3. Ziessman HA, O'Malley JP, Thrall JH, et al. Nuclear Medicine: The Requisites. 4th ed. Philadelphia, PA: Elsevier Saunders; 2014.

4. Sohaib SA, Cook G, Allen SD, et al. Comparison of whole-body MRI and bone scintigraphy in the detection of bone metastases in renal cancer. $\mathrm{Br} J$ Radiol. 2009;82:632-639. 